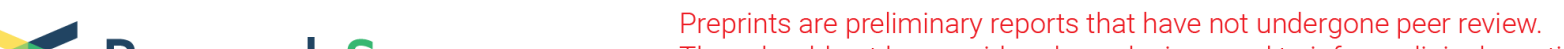 $\begin{array}{ll}\text { Research Square } & \begin{array}{l}\text { They should not be considered conclusive, used to inform clinical practice, } \\ \text { or referenced by the media as validated information. }\end{array}\end{array}$
}

\section{The DAWN study of dorzagliatin as add-on therapy to metformin in patients with type 2 diabetes: a randomized, double-blind, placebo-controlled, phase 3 trial}

\section{Li Chen ( $\square$ lichen@huamedicine.com )}

Hua Medicine (Shanghai) Ltd. https://orcid.org/0000-0002-6192-3752

\section{Wenying Yang}

China-Japan Friendship Hospital

\section{Dalong Zhu}

Nanjing University Medical School Affiliated Nanjing Drum Tower Hospital

\section{Xiaoying Li}

Department of Endocrinology and Metabolism, Fudan Institute of Metabolic Diseases, Zhongshan Hospital, Fudan University, Shanghai 200032

\section{Shenglian Gan}

The First People's Hospital of Changde City

\section{Xiaolin Dong}

Jinan Central Hospital Affiliated to Shandong University

\section{Junping Su}

Cangzhou People's Hospital

\section{Wenhui Li}

Peking Union Medical College Hospital, Department of Endocrinology

\section{Hongwei Jiang}

Henan University of Science and Technology Affiliated First Hospital

\section{Wenjuan Zhao}

The Affiliated Hospital of Qingdao University

\section{Minxiu Yao}

Qingdao Central Hospital

\section{Weihong Song}

Chenzhou No 1 People's Hospital

\section{Yibing Lu}

Nanjing Medical University Second Affiliated Hospital

\section{Xiuzhen Zhang}

Tongji Hospital Affiliated to Tongji University

\section{Huifang Li}


The First Affiliated Hospital of Kunming Medical University

\section{Guixia Wang}

Department of Endocrinology, The First Hospital of Jilin University

\section{Wei Qiu}

Huzhou Central Hospital

\section{Guoyue Yuan}

Affiliated Hospital of Jiangsu University

\section{Jianhua Ma}

Nanjing First Hospital

\section{Wei Li}

The Affiliated Hospital of Xuzhou Medical University

\section{Ziling Li}

Inner Mongolia Baogang Hospital

\section{Xiaoyue Wang}

The First People's Hospital of YueYang

\section{Jiao'e Zeng}

Jingzhou Central Hospital

\section{Zhou Yang}

JiangXi PingXiang People's Hospital

Jingdong Liu

Jiangxi Provincial People's Hospital

\section{Yongqian Liang}

The First People's Hospital of Shunde

\section{Song Lu}

Chongqing General Hospital

\section{Huili Zhang}

Qinghai University Affiliated Hospital

\section{Hui Liu}

Luoyang Central Hospital

\section{Ping Liu}

General Hospital of Ningxia Medical University

\section{Kuanlu Fan}

The General Hospital of Xuzhou City Mining Group

\section{Xiaozhen Jiang}

Shanghai Pudong New Area People's Hospital

\section{Yufeng Li}

Beijing Friendship Hospital pinggu Campus, Captital Medical University

\section{Qing Su}

Xinhua Hospital affliated to Shanghai Jiaotong University Medical School 


\section{Tao Ning}

Inner Mongolia Baotou City Central Hospital

\section{Huiwen Tan}

Sichuan University West China Hospital

\section{Zhenmei An}

Sichuan University West China Hospital

\section{Zhaoshun Jiang}

PLA joint logistic support force 960 hospital

\section{Lijun Liu}

Yiyang Central Hospital

\section{Zunhai Zhou}

Tongji University Affilliated Yangpu Hospital

\section{Qiu Zhang}

First Affiliated Hospital of Anhui Medical University

\section{Xuefeng Li}

Taihe Hospital

\section{Zhongyan Shan}

Department of Endocrinology and Metabolism, Institute of Endocrinology, First Affiliated Hospital,China Medical University,

\section{Yaoming Xue}

Southern Medical University Nanfang Hospital

\section{Hong Mao}

The Central Hospital of Wuhan

\section{Lixin Shi}

The First Hospital of Guiyang Medical School, Endocrinology

\section{Shandong Ye}

Anhui Provincial Hospital

\section{Xiaomei Zhang}

The First Hospital Affiliated to Bengbu Medical College, Department of Endocrinology

\section{Jiao Sun}

Huadong Hospital Affiliated to Fudan University

\section{Tao Yang}

The First Affiliated Hospital of Nanjing Medical University https://orcid.org/0000-0001-6375-3622

\section{Feng Li}

Jining No 1 People's Hospital

Jingna Lin

Tianjin People's Hospital Tianjin Union Medical Center

\section{Zhinong Zhang}

Qiqihar First Hospital 


\section{Ying Zhao}

Jilin Central General Hospital

\section{Ruonan Li}

Yunnan Province Third People's Hospital

\section{Xiaohui Guo}

Peking University First Hospital

\section{Qi Yao}

Ningbo City First Hospital

\section{Weiping Lu}

Huai'An First People's Hospital, Nanjing Medical University

\section{Shen Qu}

Tongji University Tenth People's Hospital

\section{Hongmei Li}

China Meitan General Hospital

\section{Liling Tan}

The First Affiliated Hospital of Hainan Medical University

\section{Wenbo Wang}

Peking University Shougang Hospital

\section{Yongli Yao}

Department of Endocrinology, Qinghai Provincial People's Hospital

\section{Daoxiong Chen}

Hainan General Hospital

\section{Yulan Li}

Liuzhou People's Hospital

\section{Jialin Gao}

The First Affiliated Hospital of Wannan Medical University

\section{Wen $\mathrm{Hu}$}

The Second People's Hospital of Huai'an

\section{Xiaoqiang Fei}

Jiangsu Taizhou People's Hospital

\section{Tianfeng Wu}

Zhejiang Hospital

\section{Song Dong}

Aerospace Center Hospital

\section{Wenlong Jin}

Yanbian University Hospital

\section{Chenzhong Li}

The Third Affiliated Hospital of Southern Medical University

Dong Zhao 
Beijing Luhe Hospital Affiliated to Capital Medical University

\section{Bo Feng}

Shanghai East Hospital

\section{Yu Zhao}

Hua Medicine (Shanghai) Ltd

\section{Yi Zhang}

Hua Medicine (Shanghai) Ltd

\section{Article}

Keywords: Metformin, type-2 diabetes, dorzagliatin

Posted Date: July 26th, 2021

DOl: https://doi.org/10.21203/rs.3.rs-733332/v1

License: (c) (i) This work is licensed under a Creative Commons Attribution 4.0 International License. Read Full License

Version of Record: A version of this preprint was published at Nature Medicine on May 12th, 2022. See the published version at https://doi.org/10.1038/s41591-022-01803-5. 


\section{Abstract}

Metformin is the first-line therapy for the treatment of type 2 diabetes (T2D) through mechanism of reduction in hepatic glucose production and fasting plasma glucose. Dorzagliatin is a novel glucokinase activator (GKA) that improves glucose and insulin sensitivity which significantly reduces post meal plasma glucose. Combination of dorzagliatin with metformin would offer benefits through the synergy of two mechanisms. In this randomized, double-blind, placebo-controlled phase 3 trial (NCT03141073), the efficacy and safety of dorzagliatin add-on to metformin in T2D patients were assessed. Eligible T2D patients ( $n=767$ ) were randomly assigned in a 1:1 ratio to dorzagliatin group or placebo group add-on to metformin ( $1500 \mathrm{mg} /$ day) for a 24-week double-blind treatment, then followed by a 28-week open-label treatment with dorzagliatin in all patients. The primary efficacy endpoint was the change from baseline in the glycated hemoglobin(HbA1c) level at week 24 and the safety was assessed throughout the trial. At week 24 , the HbA1c was reduced from baseline in dorzagliatin group, superior to placebo $(-1.02 \%$ vs. $-0.36 \%$; ETD, $-0.66 \% ; 95 \% \mathrm{Cl},-0.79$ to $-0.53 ; \mathrm{P}<0.0001)$, with the effects sustained through 52 weeks. The 2hour postprandial glucose ( $2 \mathrm{hPPG}$ ) was significantly decreased in dorzagliatin group over placebo $(-98.10 \mathrm{mg} / \mathrm{dl}$ vs. $-53.46 \mathrm{mg} / \mathrm{dl}, P<0.0001)$, and the homeostasis model assessment $2-\beta($ HOMA2- $\beta$ ) and homeostasis model assessment 2-IR (HOMA 2-IR) were significantly improved at week 24 over placebo. The incidence of adverse events (AEs) was similar between the two groups during the 24 weeks. The hypoglycemia occurred in $4(1.0 \%)$ out of 382 patients in dorzagliatin group during the 52 weeks. No severe hypoglycemia events were reported. In T2D patients with inadequate glycemic control by metformin alone, dorzagliatin demonstrated fast onset and sustained glycemic control with a good safety and tolerability profile for 52 weeks.

\section{Introduction}

The prevalence of type 2 diabetes continues increasing worldwide including China. ${ }^{1-4}$ Because of ongoing deterioration of the $\beta$-cell function and insulin sensitivity, the progressive diabetic disorder necessitates the add-on therapy to maintain appropriate glucose level. ${ }^{5,6}$ As recommended in various guidelines, metformin is the standard and preferred first-line treatment for type 2 diabetes which requires add-on therapy after its loss of glycemic control. ${ }^{7,8}$ To date, various drugs are available as add-on options, but the continued decline of $\beta$-cell function is still the unmet need of type 2 diabetes patients. In this regard, pharmacological activator of the glucokinase could provide a new alternative. ${ }^{9,10}$

Glucokinase activators (GKAs) have been evaluated in combination with metformin in several clinical trials. Dual-acting partial GKA (AZD 1656 and PF-04937319), and liver-acting full GKA (TTP399) showed glucose reduction efficacy in the phase 2 trials and are currently under clinical development. ${ }^{11-}$

${ }^{13}$ Dorzagliatin has different mechanism of action as the dual-acting full GKA, and takes effects in both pancreas and liver with the increase of glucokinase enzymatic catalytic capacity, which in turn repairs the declined glucose sensitivity in type 2 diabetes patients. ${ }^{14,15}$ 
Dorzagliatin is an allosteric glucokinase activator that regulates the pancreatic hormone secretion of insulin, glucagon-like peptide-1 and glucagon, ${ }^{16-18}$ while synchronizes the glucose and hormonal signal in the liver that controls the hepatic glucose storage and production. While the reduction of GK expression in pancreas and liver in type 2 diabetes patients is an underlying cause of impaired glucose and insulin sensitivty, ${ }^{19}$ that dorzagliatin improves the early-phase insulin secretion and whole-body glucose disposition in type 2 diabetes patients offers an opportunity to address the unmet medical needs. ${ }^{15}$ Dorzagliatin reduced the glycated hemoglobin ( $\mathrm{HbA} 1 \mathrm{c})$ effectively with low risk of hypoglycemia, which led to a remarkable improvement in the homeostatic control rate $(\mathrm{HbA} 1 \mathrm{c}$ of less than $7.0 \%$ and without hypoglycemia)in the phase 3 trial in Chinese type 2 diabetes patients (SEED trial), and the sustained improvement in disposition index after drug treatment in a phase 2 trial. ${ }^{15}$

As a novel antidiabetic agent, dorzagliatin may offer a new solution to type 2 diabetes patients with insufficient glycemic control by metformin monotherapy. Here, we report the findings of a phase 3 clinical trial (DAWN) designed to investigate the efficacy and safety, and effects on $\beta$-cell function of dorzagliatin through combination with metformin in Chinese type 2 diabetes patients.

\section{Results}

\section{Patients}

Patients were recruited from Oct 11, 2017 to Aug 30, 2019, with the last patient visit conducted on Aug 31, 2020. Of the 1721 patients screened, 767 eligible patients were randomly assigned to one of the two treatment groups; 382 were assigned to receive dorzagliatin $75 \mathrm{mg}$ twice a day plus metformin $1500 \mathrm{mg}$ daily, and 385 were assigned to receive placebo plus metformin 1500 mg daily (Fig. 1b).766 patients took at least one dose of study drug, 751 patients were included in the full analysis set and 766 patients were in the safety set. 692 patients completed 24 weeks of treatment (349 [91.4\%] in the dorzagliatin plus metformin group and 343 [89.1\%] in the placebo plus metformin group). Subsequently, 646 patients completed 52 weeks of treatment - $330(86.4 \%)$ in the dorzagliatin plus metformin group and $316(82.1 \%)$ in the placebo plus metformin group (Fig. 1b). In total, 33 patients in the dorzagliatin plus metformin group and 42 in the placebo plus metformin group discontinued treatment in 24-week double-blind treatment period, and 19 patients in the dorzagliatin plus metformin group and 27 in the placebo plus metformin group discontinued treatment in extended 28-week open-label treatment period. The demographic and baseline characteristics were similar between the two groups (Table 1). 
Table 1. Baseline Characteristics of the Patients. *

\begin{tabular}{|c|c|c|}
\hline Characteristic & $\begin{array}{c}\text { Dorzagliatin+Metformin } \\
(\mathrm{N}=382)\end{array}$ & $\begin{array}{c}\text { Placebo+Metformin } \\
(\mathrm{N}=385)\end{array}$ \\
\hline Age - yr & $54.6 \pm 10.0$ & $54.4 \pm 9.2$ \\
\hline Male sex - n(\%) & $245(64.1)$ & $230(59.7)$ \\
\hline Duration of diabetes - mth & $73.7 \pm 58.5$ & $69.4 \pm 53.0$ \\
\hline Body weight - kg & $70.8 \pm 11.4$ & $71.5 \pm 12.2$ \\
\hline Body-mass index§- $\mathrm{kg} / \mathrm{m}^{2}$ & $25.7 \pm 3.0$ & $26.1 \pm 3.2$ \\
\hline \multicolumn{3}{|l|}{ Blood pressure - mm Hg } \\
\hline Systolic & $125.7 \pm 12.5$ & $125.3 \pm 12.3$ \\
\hline Diastolic & $79.9 \pm 8.0$ & $80.3 \pm 8.1$ \\
\hline Glycated hemoglobin - \% & $8.3 \pm 0.6$ & $8.3 \pm 0.6$ \\
\hline Fasting plasma glucose - mg/dl & $175.9 \pm 28.9$ & $179.1 \pm 30.0$ \\
\hline 2-hour postprandial glucose - mg/dl & $338.8 \pm 55.0$ & $343.4 \pm 54.0$ \\
\hline Fasting C peptide - ng/mL & $1.520 \pm 0.5545$ & $1.606 \pm 0.6477$ \\
\hline HOMA2- $\beta$ & $31.11 \pm 11.821$ & $31.43 \pm 12.651$ \\
\hline HOMA2-IR & $1.3853 \pm 0.5150$ & $1.4918 \pm 0.7034$ \\
\hline Medical history - n(\%) & $363(95.0)$ & $366(95.1)$ \\
\hline Metabolic and nutritional diseases & 229 ( 59.9) & $213(55.3)$ \\
\hline Hyperlipemia & $141(36.9)$ & $142(36.9)$ \\
\hline Dyslipidemia & $43(11.3)$ & $36(9.4)$ \\
\hline Hypertriglyceridemia & $14(3.7)$ & $7(1.8)$ \\
\hline Low HDL cholesterolemia & $7(1.8)$ & $8(2.1)$ \\
\hline Hypercholesteremia & $5(1.3)$ & $3(0.8)$ \\
\hline Vascular and lymphatic diseases & 183 ( 47.9$)$ & $194(50.4)$ \\
\hline Liver and biliary system diseases & $135(35.3)$ & $147(38.2)$ \\
\hline Infectious and infectious diseases & $98(25.7)$ & 105 ( 27.3) \\
\hline Neurological diseases & 99 ( 25.9) & $86(22.3)$ \\
\hline Kidney and urinary system diseases & $92(24.1)$ & 111 ( 28.8) \\
\hline Heart organ disease & $74(19.4)$ & $67(17.4)$ \\
\hline Neurological diseases & 99 ( 25.9) & $86(22.3)$ \\
\hline ALT-U/L & $23.0 \pm 13.7$ & $24.3 \pm 13.9$ \\
\hline AST-U/L & $19.8 \pm 8.6$ & $21.0 \pm 9.2$ \\
\hline TBiill- umol/L & $10.9 \pm 4.80$ & $11.0 \pm 4.52$ \\
\hline $\mathrm{TG}-\mathrm{mmol} / \mathrm{L}$ & $1.88 \pm 1.30$ & $1.90 \pm 1.13$ \\
\hline $\mathrm{TC}-\mathrm{mmol} / \mathrm{L}$ & $4.51 \pm 0.89$ & $4.54 \pm 0.86$ \\
\hline LDL-C - mmol/L & $2.47 \pm 0.78$ & $2.47 \pm 0.74$ \\
\hline HDL-C - mmol/L & $1.22 \pm 0.28$ & $1.23 \pm 0.28$ \\
\hline $\mathrm{eGFR}-\mathrm{mL} / \mathrm{min} / 1.73 \mathrm{~m}^{2} \dagger$ & $99.6 \pm 19.3$ & $98.2 \pm 19.0$ \\
\hline
\end{tabular}

* Plus-minus values are means \pm SD. To convert the values for plasma glucose to millimoles per liter, divided by 18 .

$\S$ Body-mass index (BMI) is the weight in kilograms divided by the square of the height in meters. \# The incidence was calculated according to the total number of female.

† eGFR was calculated in 766 patients who were in the safety set population. 


\section{Efficacy}

The $\mathrm{HbA} 1 \mathrm{c}$ level was reduced from baseline by 1.02 percentage points in the dorzagliatin plus metformin group at week 24, whereas the level decreased by 0.36 percentage points in the placebo plus metformin group (estimated treatment difference, -0.66 percentage points; $95 \% \mathrm{Cl},-0.79$ to -0.53 ; $\mathrm{P}<0.001$ ) (Fig. $2 \mathrm{a}$ ). The results demonstrated the superiority of dorzagliatin plus metformin to placebo plus metformin in the reduction of $\mathrm{HbA} 1 \mathrm{c}$. The effects of dorzagliatin plus metformin in reducing fasting plasma glucose and 2-hour postprandial glucose levels at week 24 compared to placebo plus metformin were also shown. At week 24, a greater reduction in the 2-hour postprandial glucose from baseline was observed with dorzagliatin plus metformin than with placebo plus metformin $(-98.10 \mathrm{mg}$ per deciliter vs. $-53.46 \mathrm{mg}$ per deciliter; estimated treatment difference, $-44.64 \mathrm{mg}$ per deciliter; $95 \% \mathrm{Cl},-53.28$ to -35.82 , ; $\mathrm{P}<0.0001$ ) (Fig. $2 \mathrm{~b})$. The fasting plasma glucose level was reduced in the dorzagliatin plus metformin group over the placebo plus metformin group at week 24 (-12.06 mg per deciliter vs. $-5.22 \mathrm{mg}$ per deciliter; estimated treatment difference, $-6.84 \mathrm{mg}$ per deciliter; $95 \% \mathrm{Cl},-12.06$ to $-1.62, \mathrm{P}<0.01$ ) (Table 2).

\section{Changes in Efficacy Endpoints From Baseline to Week 24 and Week 52}

\begin{tabular}{|c|c|c|c|c|}
\hline Endpoints & $\begin{array}{l}\text { Dorzagliatin+ } \\
\text { Metformin } \\
(\mathrm{N}=376)\end{array}$ & $\begin{array}{l}\text { Placebo+ } \\
\text { Metformin } \\
(\mathrm{N}=375)\end{array}$ & $\begin{array}{l}\text { Estimated Treatment } \\
\text { Difference, Dorzagliatin } \\
\text { vs. Placebo }(95 \% \mathrm{CI}) \\
\end{array}$ & P Value \\
\hline \multicolumn{5}{|l|}{ hemoglobin *- \% } \\
\hline $\begin{array}{l}\text { an change from baseline to week } \\
\text { ostprandial glucose } *_{-} \mathrm{mg} / \mathrm{dl}\end{array}$ & $-1.02 \pm 0.047$ & $-0.36 \pm 0.048$ & $-0.66(-0.79,-0.53)$ & $<0.0001$ \\
\hline an change from baseline to week & $-98.10 \pm 3.31$ & $-53.46 \pm 3.31$ & $-44.64(-53.28,-35.82)$ & $<0.0001$ \\
\hline \multicolumn{5}{|l|}{ गlasma glucose - mg/dl } \\
\hline ıean change from baseline to week & $-12.06 \pm 1.91$ & $-5.22 \pm 1.91$ & $-6.84(-12.06,-1.62)$ & $<0.01$ \\
\hline \multicolumn{5}{|c|}{ e rate (glycated hemoglobin $<7.0 \%)^{\dagger}{ }_{-} \%$} \\
\hline $\begin{array}{l}\text { Ige of patients achieving target at } \\
\text { asis model assessment } 2-\beta\end{array}$ & 44.4 & 10.7 & $7.60(5.05,11.42)$ & $<0.0001$ \\
\hline ıean change from baseline to week & $3.82 \pm 0.71$ & $1.40 \pm 0.70$ & $2.43(0.59,4.26)$ & $<0.01$ \\
\hline $\begin{array}{l}\text { nated mean change from baseline } \\
52 \| \\
\text { asis model assessment } 2 \text {-IR }\end{array}$ & $1.96 \pm 12.21$ & $5.50 \pm 14.38$ & NA & NA \\
\hline ıean change from baseline to week & $-0.17 \pm 0.03$ & $-0.09 \pm 0.03$ & $-0.08(-0.15,-0.01)$ & $<0.05$ \\
\hline $\begin{array}{l}\text { nated mean change from baseline } \\
52 \|\end{array}$ & $-0.17 \pm 0.39$ & $-0.18 \pm 0.49$ & NA & NA \\
\hline
\end{tabular}


* Plus-minus values are estimated LS means \pm SE. LS mean differences and corresponding 95\% confidence interval (CI) were estimated using a mixed model for repeated measures in the full-analysis set.

† Response rate was estimated in the full-analysis set, with the use of the data imputed by last observation carried forward. Estimated treatment difference is represented as the odds ratio and 95\%CI using logistic regression.

‡ Plus-minus values are estimated LS means \pm SE. LS mean differences were calculated from an analysis of covariance in the full-analysis set, with the use of the data imputed by last observation carried forward.

\| Plus-minus values are arithmetic means \pm SD.

SE: standard error; SD: standard deviation; NA: not available; LS: least-squares; CI: confidence interval.

Results showed that $44.4 \%$ of the patients in the dorzagliatin plus metformin group, as compared with $10.7 \%$ in the placebo plus metformin group at week 24, achieved a $\mathrm{HbA} 1 \mathrm{c}$ response rate (a $\mathrm{HbA} 1 \mathrm{c}$ level of less than 7.0\%) (odds ratio, 7.60; 95\% Cl, 5.05 to 11.42, $\mathrm{P}<0.0001$ ) (Table 2). The similar percentage of patients (40.6\%) was achieved in the dorzagliatin plus metformin group since week 8 , over that in the placebo plus metformin group (5.6\%), and sustained until week 24 (Supplementary Table 4). The composite endpoint of response rate (the HbA1c of less than $7.0 \%$, without hypoglycemia and no weight gain) in the dorzagliatin plus metformin group achieved $26.60 \%$ compared with $7.70 \%$ in the placebo plus metformin group at week 24 (odds ratio, 4.31; 95\% Cl, 2.75 to 6.75, $\mathrm{P}<0.0001$ ) (Fig. 2c).

Homeostasis model assessment 2- $\beta$ change from baseline was improved at week 24 in the dorzagliatin plus metformin group compared with the placebo plus metformin group (3.82 with dorzagliatin vs. 1.40 with placebo; estimated treatment difference, 2.43; 95\% Cl, 0.59 to 4.26, $\mathrm{P}<0.01$ ) (Table 2 and Fig. 2d). Homeostasis model assessment 2-IR change from baseline was improved as well at week 24 in the dorzagliatin plus metformin group compared with the placebo plus metformin group $(-0.17$ with dorzagliatin vs. -0.09 with placebo; estimated treatment difference, $-0.08 ; 95 \% \mathrm{Cl},-0.15$ to $-0.01, \mathrm{P}<0.05)$ (Table 2 and Fig. 2e).

During the extended 28-week open-label treatment, homeostasis model assessment 2-IR continued to decrease numerically in dorzagliatin plus metformin group (Table 2). The effect of lowering HbA1c level in the dorzagliatin plus metformin group started at week 4 and a significantly greater reduction compared to placebo maintained until the end of the 24-week treatment period. Moreover, the effect of reducing the $\mathrm{HbA1c}$ level in the dorzagliatin plus metformin group sustained from week 24 to week 52 . The decrease of the $\mathrm{HbA} 1 \mathrm{c}$ level was also observed in the placebo plus metformin group after switching to dorzagliatin plus metformin treatment at week 24 to week 52 (Fig. 2f).

\section{Safety}

During the 24-week double-blind treatment period, the percentage of patients with adverse events and serious adverse events was similar in the dorzagliatin plus metformin group and placebo plus metformin group (Table 3). Most of the adverse events were mild, resolved, and were considered to be unrelated to the study drugs by investigators. There was no clustering of serious adverse events (Supplementary 
Table 9). No serious adverse events were considered as related to dorzagliatin or placebo by investigators. Adverse events that occurred in at least $5 \%$ of the patients in two groups were listed in Table 3. During the open-label treatment period, both adverse events and serious adverse events remain as the same type and incidence rate as that has occurred in the double-blind treatment period. During the double-blind treatment period, BMI decreased in both groups, and no statistical difference was observed between the two groups, suggesting that dorzagliatin treatment did not cause weight gain in drug-naïve type 2 diabetes patients in this study. There was no statistical difference in blood pressure between the two groups (Table 4).

Table 3. Adverse Events and Hypoglycemic Events in 24-week Double-blind Treatment Period.* Event

\begin{tabular}{cc}
$\begin{array}{c}\text { Dorzagliatin+Metformin } \\
\mathbf{( N = 3 8 2 )}\end{array}$ & $\begin{array}{c}\text { Placebo+Metformin } \\
\mathbf{( N = 3 8 4 )}\end{array}$ \\
No. of Patients (\%) & No. of Patients (\%) \\
\hline $299(78.3)$ & $278(72.4)$ \\
$53(13.9)$ & $36(9.4)$ \\
$19(5.0) \dagger$ & $17(4.4)$ \\
$1(0.3)$ & $3(0.8)$ \\
$3(0.8)$ & $4(1.0)$
\end{tabular}

Any adverse events

Adverse events related to Dorzagliatin\#

$53(13.9)$

$36(9.4)$

Any serious adverse events

$1(5.3)$

$3(0.8)$

Adverse events leading to drug discontinuation $\uparrow$

$3(0.8)$

$4(1.0)$

Adverse events in $\geq 5 \%$ of patients $\|$

Upper respiratory tract infection

$54(14.1)$

$52(13.5)$

Hyperlipidemia

$52(13.6)$

$33(8.6)$

Protein urine present

30 (7.9)

$30(7.8)$

Hyperuricemia

37 (9.7)

14 (3.6)

Hypertriglyceridemia

$21(5.5)$

$11(2.9)$

Adverse events in $\geq 5 \%$ of patients related to the study drug $\S$

Upper respiratory tract infection

0

$2(0.5)$

0

$3(0.8)$

$7(1.8)$

Hypertriglyceridemia

Any hypoglycemia

Severe hypoglycemia

Clinically significant hypoglycemia (Blood glucose level $<3.0$ $\mathrm{mmol} / \mathrm{L}$ )

$\begin{array}{cc}0 & 0 \\ 3(0.8) & 0\end{array}$

*Adverse events and serious adverse events that occurred in the 24-week double-blind treatment period among patients in the safety population are included in the table and presented with their preferred terms in the Medical Dictionary for Regulatory Activities, version 23.0. Data are for patients who received at least one dose of a study drug and include events that occurred during treatment or within 7 days after the last receipt of a study drug.

\#Possibly or very likely related to dorzagliatin, as assessed by investigators.

TSerious adverse events that leading to drug discontinuation are not included.

†During 24-week double-blind treatment period, one patient in the dorzagliatin+metformin group died (confirmed by the external drug safety committee as sudden death). The patient had type 2 diabetes and established cardiovascular disease, and the event was considered as unlikely related to dorzagliatin by both the investigator and the sponsor.

Page $11 / 22$ 
\|Adverse events in at least $5 \%$ of patients in the dorzagliatin+metformin group or the placebo+metformin group were listed in the table.

§Adverse events in at least $5 \%$ of patients related to the dorzagliatin were defined as adverse events that were deemed by the investigators to be very likely, or probably related to the dorzagliatin or placebo.

Table 4. Changes in Selected Labs and Vital Signs (Safety Set).

\begin{tabular}{lc|c|c|c} 
& \multicolumn{2}{c|}{$\begin{array}{c}\text { Dorzagliatin+Metformin } \\
(\mathbf{N = 3 8 2 )}\end{array}$} & \multicolumn{2}{c}{$\begin{array}{c}\text { Placebo+Metformin } \\
(\mathbf{N = 3 8 4 )}\end{array}$} \\
& Week 24 & Week 52 & Week 24 & Week 52 \\
\hline Change in body weight $-\mathrm{kg}$ & $-0.19 \pm 2.270$ & $-0.50 \pm 2.021$ & $-0.45 \pm 2.156$ & $-0.30 \pm 2.220$ \\
Change in body mass index- $\mathrm{kg} / \mathrm{m}^{2}$ & $-0.065 \pm 0.8164$ & $-0.179 \pm 0.7430$ & $-0.170 \pm 0.7882$ & $-0.114 \pm 0.8371$ \\
Change in systolic blood pressure- mm Hg & $-0.18 \pm 12.175$ & $-0.20 \pm 12.936$ & $-1.20 \pm 12.644$ & $2.02 \pm 12.522$ \\
Change in diastolic blood pressure- $\mathrm{mm} \mathrm{Hg}$ & $0.16 \pm 8.465$ & $0.00 \pm 9.081$ & $-1.42 \pm 7.930$ & $-0.08 \pm 8.000$
\end{tabular}

Plus-minus values are arithmetic means \pm SD.

The clinically significant hypoglycemia (blood glucose level $<54 \mathrm{mg}$ per deciliter) occurred in $4(1.0 \%)$ out of 382 patients in the dorzagliatin plus metformin group during the 52 weeks (Table 3 and Supplementary Table 8). No severe hypoglycemia episodes were reported.

\section{Discussion}

The synergistic effect of metformin and dorzagliatin was evidenced in the DAWN trial. In those patients whose glycemic control has failed with full dose of metformin, quick on-set in the HbA1c reduction was observed and sustained during the 24-week double-blind treatment (Fig. 2a), with significant reduction in the postprandial glucose (Fig. $2 \mathrm{~b}$ ) and the fasting plasma glucose compared with placebo (Table 2). The characteristics of the patients who enrolled into the DAWN study were observed at baseline. The duration of disease was about 6 years, the baseline $\mathrm{HbA} 1 \mathrm{c}$ level was about $8.3 \%$ and the body-mass index about $26 \mathrm{~kg} / \mathrm{m}^{2}$, accompanied by high 2-hour postprandial glucose about $340 \mathrm{mg} / \mathrm{dl}$ (Table 1). It is likely that the patients still suffered from impaired glucose sensitivity and decreased glucokinase expression in both pancreas and liver over time, which may result in reduced hepatic glucose uptake and increased hepatic glucose output. Dorzagliatin repaired these defects and achieved effective control in the fasting plasma glucose and postprandial glucose accompanied with the improvement of $\beta$-cell function and reduction of insulin resistance indicated by homeostasis model assessment 2- $\beta$ and homeostasis model assessment 2-IR as shown in Fig. 1d and 1e. Mechanistic studies need to be conducted further to elucidate the mechanism of action of dorzagliatin and advance the application of glucokinase-based therapy. Additional benefits over other oral antidiabetic agents were observed in the dorzagliatin-treated group in terms of the improved composite endpoint of response rate and the low incidence of hypoglycemia when combined with metformin (Fig. 1c and Table 3). 
We found that dorzagliatin add-on to metformin was well-tolerated and had a good safety profile during the entire 52-week treatment period, with no occurrence of drug-related serious adverse events or severe hypoglycemia in the dorzagliatin plus metformin group. The incidence of adverse events was similar between the dorzagliatin plus metformin and the placebo plus metformin group. Clinically significant hypoglycemia only occurred in 4 patients (1.0\%) treated with dorzagliatin plus metformin and was mild in nature.

Metformin has been used as a first-line therapy for the treatment of type 2 diabetes for over 60 years. It is commonly agreed that its anti-hyperglycemic effect primarily comes from the reduction of hepatic glucose output which antagonizes the glucagon effect. ${ }^{20}$ The mode of action of metformin has been studied in three major signaling pathways including the activation of AMP-activated protein kinase and inhibition of fructose-1,6-bisphosphatase-1. ${ }^{21,22}$ The loss of efficacy in type 2 diabetes patients during metformin treatment is associated with the deterioration of $\beta$-cell function to secret early-phase insulin. ${ }^{23}$ In fact, the reduction of $\beta$-cell mass together with impaired secretory function are recognized as the key pathogenic factors in type 2 diabetes recently. ${ }^{24,25}$ Therapeutic glucokinase activator dorzagliatin showed its effects in rescuing $\beta$-cell mass in the diabetes rat and improvement of the early-phase insulin secretion which led to improved disposition index and composite endpoint in type 2 diabetes patients. ${ }^{14,15,26}$ In addition, results showed that the efficacy sustained during the 28-week open-label treatment period in the dorzagliatin group.

For safety profiles, the baseline status of subjects in the DAWN study reflected the characteristics of Chinese patients with type 2 diabetes that had high rate of complications, such as about $55 \%$ of patients with dyslipidemia related diseases, about $48 \%$ with vascular and lymphatic diseases, about $35 \%$ with liver and biliary system diseases, about $25 \%$ with kidney and urinary system diseases at screening and with high level of triglyceride (about $1.9 \mathrm{mmol} / \mathrm{L}$ at baseline) (Table 1). After 52 weeks of dorzagliatin treatment, the results in DAWN study showed similar trend as SEED study and suggested that long-term use of dorzagliatin add-on to metformin did not cause weight gain or increase blood pressure, and it did not increase the risks of abnormal blood lipid, abnormal liver function or abnormal renal function.

The limitation of this trial is that it is focused on Chinese type 2 diabetes population. Meanwhile, a clinical pharmacology study conducted in the US showed that co-administration of dorzagliatin and metformin in a small group of Caucasian type 2 diabetes patients can improve the glycemic control (NCT02597400). Further clinical trials are needed to evaluate this combination therapy in larger cohorts. In addition, the combined treatment of dorzagliatin and metformin offered additional benefits to the patients in the improvement of glucose and insulin sensitivity, which may be in favor of the early combination treatment for diabetes control reported in other studies. ${ }^{27}$

In summary, this DAWN trial showed that dorzagliatin add-on to metformin can provide effective glycemic control with good safety profiles in type 2 diabetes patients inadequately controlled with metformin. Improvement in the glucose sensitivity and insulin resistance has become additional benefits of this new treatment regimen. 


\section{Methods}

\section{Trial Design}

We conducted this phase 3 trial (DAWN) from Oct 11, 2017 to Aug 31, 2020 at 73 sites in China, in Chinese type 2 diabetes patients with insufficiently controlled blood glucose on metformin. The DAWN trial included a 2-week screening period, a 4-week single-blind run-in period, a 24-week double-blind placebo-controlled treatment period followed by an extended 28-week open-label treatment period, and a 1-week follow-up period (Fig. 1a). During the single-blind placebo run-in period, patients were treated with metformin at $1500 \mathrm{mg}$ per day as basic therapy. Patients were randomly assigned in a 1:1 ratio to receive either dorzagliatin twice a day plus daily metformin $(1500 \mathrm{mg})$ or placebo twice a day plus daily metformin (1500 mg) for a 24-week double-blind period, on a background of a diet and exercise regimen. Then all patients received dorzagliatin twice a day plus daily metformin $(1500 \mathrm{mg})$ treatment for a 28week open-label period. During the open-label period, patients assigned to dorzagliatin plus metformin continued their treatment unchanged, whereas those assigned to placebo plus metformin switched from the placebo to dorzagliatin plus metformin. After the 52-week treatment, all patients were observed for 1 week for safety evaluation.

Patients were recruited at the study site by investigators. Patients were required to sign the informed consent form before enrollment. After providing written informed consent, patients were screened for eligibility. Patients who were 18 to 75 years of age, diagnosed with type 2 diabetes, and on diet and exercise interventions, who were receiving stable daily doses of metformin (1500 mg per day at minimum) at least 12 weeks before screening, and who had a HbA1c level of 7.5 to $10.0 \%$, and a bodymass index of 18.5 to 35.0 kilogram per square meters at screening were eligible for enrollment. Key exclusion criteria were any previous antidiabetic treatment other than metformin within 12 weeks, previous insulin treatment for more than 30 days within 1 year before screening, severe hypoglycemia without cause within 3 months, or frequent hypoglycemia occurred more than 3 times within 1 month before screening, fasting C-peptide level of less than $0.81 \mathrm{ng}$ per milliliter at screening, and a history of type 1 diabetes. Other exclusion criteria were the major cardio-cerebrovascular diseases within 6 months before screening; unstable or rapidly progressive kidney disease; active liver diseases; psychiatric disease; any type of malignancy; an alanine or aspartate transaminase level 2.5 times greater than the upper limit of the normal range, or serum total bilirubin level 1.5 times greater than the upper limit of the normal range; eGFR $<60 \mathrm{ml} / \mathrm{min} / 1.73 \mathrm{~m}^{2}$ at screening. Complete lists of inclusion, exclusion, and randomization criteria are provided in the protocol, available in the supplemental material.

\section{PrOCEDURES}

At week 3 in the 4-week run-in period, patients were evaluated before randomization to confirm the eligibility. Eligibility criteria included a $\mathrm{HbA} 1 \mathrm{c}$ level of 7.5 to $10.0 \%$, and a fasting plasma glucose level of 7.0 to $13.3 \mathrm{mmol}$ per liter (126 to $239 \mathrm{mg} / \mathrm{dl}$ ) at randomization. Diet and exercise counseling was provided during the entire experiment. Randomization and drug dispense were performed with an 
interactive web response system (IWRS) (Balance, MedData Solution). The random allocation sequences were generated by the IWRS. A stratified randomization method with the permuted block randomization algorithm was used. The blocks were dynamically allocated to each site and stratum from the randomization list. A unique identification number (drug box serial number) was provided by the study medication packing provider and marked on the label of drug box. By central randomization, the randomization codes were generated by IWRS system based on randomization factors (T2DM disease duration $\leq 3$ years or $>3$ years and $\mathrm{HbA} 1 \mathrm{c}$ level $\leq 8.5 \%$ or $>8.5 \%$ at randomization) and the block size. Then, the randomization codes along with the corresponding drug box numbers were provided to subjects who meet the randomization criteria at each visit.

Investigators enrolled participants and used IWRS to assign participants to interventions. Double-blind method was used in the study. The random allocation sequences were concealed from patients, investigators and all other study members. Placebo tablets were designed with the size, color, smell and appearance same as the active drug tablets. Blinding was maintained until the end of week 24 . All doses of dorzagliatin $75 \mathrm{mg}$ tablets and placebo tablets were administered orally twice daily, as single tablets.

\section{EFFICACY Endpoints}

The primary efficacy endpoint was the change in the HbA1c level from baseline to week 24. Key secondary efficacy endpoints included the changes in the 2-hour postprandial glucose level, fasting plasma glucose level from baseline to week 24, and the HbA1c level at each visit (except week 24); the $\mathrm{HbA} 1 \mathrm{c}$ response rate (the percentage of patients who reached a $\mathrm{HbA} 1 \mathrm{c}$ level of less than $7.0 \%$ ) at week 24 . Additional efficacy endpoints included the change in the homeostasis model assessment 2- $\beta$ (an index for $\beta$-cell function) as well as the homeostasis model assessment 2 for insulin resistance (IR) from baseline. A full list of other efficacy endpoints is provided in the statistical analysis plan as the supplemental material.

\section{SAFETY ENDPOINTS}

Safety assessments were completed at every study visit. The blood and urine were sampled at each visit. Adverse events, serious adverse events were assessed throughout the trial (definitions are provided in the protocol as a supplemental material). Hypoglycemic episodes were classified according to the American Diabetes Association definitions (see the protocol provided as a supplemental material). Vital signs and clinical laboratory test results were assessed, and physical examinations were performed.

\section{TRIAL Oversight}

The trial was conducted in accordance with the principles of Declaration of Helsinki, Good Clinical Practice guidelines, and laws and regulations in China. The study protocol was amended once during study enrollment, and the important changes to methods after trial commencement and reasons were summarized in the supplemental material. The trial protocol and amendments were approved by the local ethics committees of all study sites. All patients provided written informed consent before trial entry. The 
trial was also conducted in accordance with the Chinese Diabetes Society guidelines, which require physicians to educate and strictly enforce improved exercise and dietary control as well as selfmonitoring of blood glucose (at least 2 times per week), in treating type 2 diabetes patients.

\section{Statistical Analyses}

We hypothesized that dorzagliatin would show superiority to placebo in the decrease of HbA1c level in patients after 24-week treatment. For the primary endpoint of the change from baseline in $\mathrm{HbA} 1 \mathrm{c}$ level at week 24 , we calculated that a total sample size of 750 patients would provide the trial with $95.4 \%$ power to detect a difference of 0.4 percentage points between the dorzagliatin plus metformin group and the placebo plus metformin group in a 1:1 ratio of allocation at a 2-sided significance level of 0.05 , assuming a standard deviation (SD) of 1.5 percentage points. The full analysis set included all randomized patients, who took at least one dose of study drugs and had at least one post-treatment measurement of the primary endpoint during double-blind treatment period. The safety set included all randomized patients, who took at least one dose of study drugs. Since this trial had only one confirmatory hypothesis test, i.e. the test of null hypothesis of no difference in the primary endpoint between dorzagliatin plus metformin and placebo plus metformin, there was no adjustment for multiplicity.

The primary analysis method for the primary efficacy endpoint was in the full analysis set using a mixed model for repeated measures without missing value imputation, which included treatment group, scheduled visit, interaction of treatment group with scheduled visit, pooled site, duration of diabetes ( $\leq 3$ years or $>3$ years) and $\mathrm{HbA} 1 \mathrm{c}$ baseline value as fixed effects. In the mixed model for repeated measures, data collected after initiation of prohibited antidiabetic medications (the amount and time of taking were reviewed in blind-data-review meeting and open-data-review meeting) were handled as missing data. The least-squares means of each treatment group, the estimated treatment difference between dorzagliatin plus metformin and placebo plus metformin and its $95 \%$ confidence interval $(\mathrm{Cl})$ were calculated. A sensitivity analysis was performed with the primary efficacy endpoint in the full analysis set using an analysis of covariance model with factors of treatment group, pooled site, duration of diabetes ( $\leq 3$ years or $>3$ years) and $\mathrm{HbA} 1 \mathrm{c}$ baseline value. In the analysis of covariance model, missing values were imputed using the method of last observation carried forward, which was applied after excluding data collected after initiation of prohibited antidiabetic medications. Another sensitivity analysis was performed with the primary efficacy endpoint using the same mixed model for repeated measures in the per-protocol set. The missing data of the primary efficacy endpoint due to the coronavirus disease epidemic were handled with the methods detailed in the statistical analysis plan and then a corresponding sensitivity analysis was carried out using the same method as the primary analysis.

The secondary efficacy endpoints including changes from baseline in 2-hour postprandial glucose, fasting plasma glucose levels at week 24, and the HbA1c level at each visit (except week 24) were assessed using the same mixed model for repeated measures for the primary endpoint. Another secondary efficacy endpoint of the $\mathrm{HbA} 1 \mathrm{c}$ response rate (percentage of patients who reached a $\mathrm{HbA} 1 \mathrm{c}$ level of less than $7.0 \%$ ) was estimated based on the data imputed by last observation carried forward 
approach in the full analysis set. Odds ratio and $95 \%$ confidence interval between two treatment groups were estimated using the logistic regression model with factors of treatment group, pooled site, interaction of treatment group with pooled site (interaction effect removed from the model if not significant at a 2-sided alpha level of 0.1 ), duration of diabetes ( $\leq 3$ years or $>3$ years) and baseline $\mathrm{HbA} 1 \mathrm{c}$ level. For other efficacy endpoints, such as the changes from baseline in the homeostasis model assessment $2-\beta$ and homeostasis model assessment 2 -insulin resistance were analyzed in the full analysis set using the same analysis of covariance model as described above.

The incidence of treatment-emergent adverse events that occurred between the first intake of double-blind study medications and the 7th day after the last dose of study medications was summarized by treatment group and compared between two treatment groups. The incidence of hypoglycemic events was also summarized by treatment group and compared between treatment groups. Additional details regarding the statistical analysis are provided in the statistical analysis plan as a supplemental material.

\section{Declarations}

Reporting Summary. Further information on research design is available in the Nature Research Reporting Summary linked to this article.

\section{Data availability}

We prefer not to share the data collected for our study, including individual patient data and a data dictionary defining each field in the data set.

\section{Code availability}

Commercially available software (SAS version 9.4, SAS Institute) was used for analyses.

\section{Acknowledgements}

We thank Jing Chen, Ph.D., for medical writing and editing support, funded by Hua Medicine; Yang Yang, and Lin Li (Hua Medicine), for assistance with the efficacy data; Aihua Wang (Hua Medicine), for assistance with the safety data; Xiang Liu (Hua Medicine), for assistance with the statistical analyses; and Gary Yu and Fuxing Tang, who reviewed an earlier version of the manuscript on behalf of Hua Medicine. The study is funded by Hua Medicine, and in part, by grants from the National Major Scientific and Technological Special Project for Significant New Drugs Development (2014ZX09101002004, 2018ZX09711002-012-001), Shanghai Science and Technology Innovation Action Project (14431908300, 15XD1520500, 17DZ1910200, 19431905200), Shanghai Pudong District Science and Technology Innovation Action Project (PKJ2014-S06), Shanghai Municipal Commission of Economy and Informatization Innovation Action Project (XC-ZXSJ-01-2015-02, 18XI-18).

\section{Author contributions}


Wenying Yang led the design, conduct and analysis of the clinical study, as well as the development of the manuscript. The trial was designed and overseen by authors who are employees of the sponsor (Hua Medicine) with input from selected site investigators. The site investigators collected the data, and the sponsor performed the data analyses. All the authors were involved in the interpretation of data, and vouch for the accuracy and completeness of the data and the fidelity of the trial to the protocol. The first author wrote the first draft of the manuscript with assistance from an independent medical writer funded by the sponsor. The manuscript was subsequently revised and approved by all the authors, who agreed to submit the manuscript for publication.

\section{Competing interests}

The authors declare no competing interests.

\section{References}

1. Saeedi P, et al. Global and regional diabetes prevalence estimates for 2019 and projections for 2030 and 2045: Results from the International Diabetes Federation Diabetes Atlas, 9th edition. Diabetes Res Clin Pract 2019;157:107843.

2. Yang W, et al. Prevalence of diabetes among men and women in China. N Engl J Med 2010;362:1090-101.

3. Xu Y, et al. Prevalence and control of diabetes in Chinese adults. JAMA 2013;310:948-59.

4. Li Y, et al. Prevalence of diabetes recorded in mainland China using 2018 diagnostic criteria from the American Diabetes Association: national cross sectional study.BMJ 2020;369:m997.

5. Kahn SE. The relative contributions of insulin resistance and beta-cell dysfunction to the pathophysiology of Type 2 diabetes.Diabetologia. 2003;46:3-19.

6. DeFronzo RA. From the triumvirate to the "ominous octet": a new paradigm for the treatment of type 2 diabetes mellitus. Clinical Diabetology 2009;10:101-28.

7. American Diabetes Association. Pharmacologic Approaches to Glycemic Treatment: Standards of Medical Care in Diabetes-2021.Diabetes Care 2021; 44: S111-S124.

8. Cosentino F, et al. 2019 ESC Guidelines on diabetes, pre-diabetes, and cardiovascular diseases developed in collaboration with the EASD. Eur Heart J 2020;41:255-323.

9. Grimsby J, et al. Allosteric Activators of Glucokinase: Potential Role in Diabetes Therapy.Science 2003;301:370-3.

10. Matschinsky FM.Assessing the potential of glucokinase activators in diabetes therapy.Nat Rev Drug Discov 2009;8:399-416.

11. Wilding JP, et al. Dose-ranging study with the glucokinase activator AZD1656 in patients with type 2 diabetes mellitus on metformin.Diabetes Obes Metab. 2013;15:750-9.

12. Amin NB, et al. Two dose-ranging studies with PF-04937319, a systemic partial activator of glucokinase, as add-on therapy to metformin in adults with type 2 diabetes.Diabetes Obes Metab 
2015;17:751-9.

13. Vella A, et al. Targeting hepatic glucokinase to treat diabetes with TTP399, a hepatoselective glucokinase activator.Sci Trans/ Med 2019;11:eaau3441.

14. Zhu XX, et al. Dorzagliatin (HMS5552), a novel dual-acting glucokinase activator, improves glycaemic control and pancreatic $\beta$-cell function in patients with type 2 diabetes: A 28-day treatment study using biomarker-guided patient selection.Diabetes Obes Metab 2018;20:2113-2120.

15. Zhu D, et al. Dorzagliatin monotherapy in Chinese patients with type 2 diabetes: a dose-ranging, randomised, double-blind, placebo-controlled, phase 2 study. Lancet Diabetes Endocrinol. 2018 Aug;6(8):627-636.

16. Johnson D, et al. Glucose-dependent modulation of insulin secretion and intracellular calcium ions by GKA50, a glucokinase activator.Diabetes $2007 ; 56: 1694-702$.

17. Matschinsky FM, et al.Glucokinase activators for diabetes therapy: May 2010 status report.Diabetes Care 2011;34 Suppl 2:S236-43.

18. Moede T, et al. Glucokinase intrinsically regulates glucose sensing and glucagon secretion in pancreatic alpha cells.Sci Rep 2020;10:20145.

19. Haeusler RA, et al. Decreased expression of hepatic glucokinase in type 2 diabetes. Mol Metab 2014;4:222-6.

20. Rena G, et al. The mechanisms of action of metformin.Diabetologia 2017;60:1577-1585.

21. Pernicova I, Korbonits M. Metformin-mode of action and clinical implications for diabetes and cancer.Nat Rev Endocrinol 2014;10:143-56.

22. Hunter RW, et al. Metformin reduces liver glucose production by inhibition of fructose-1-6bisphosphatase. Nat Med 2018;24:1395-1406.

23. Fonseca VA.Defining and characterizing the progression of type 2 diabetes.Diabetes Care 2009;32 Suppl 2:S151-6.

24. Meier JJ, Bonadonna RC.Role of reduced $\beta$-cell mass versus impaired $\beta$-cell function in the pathogenesis of type 2 diabetes.Diabetes Care 2013;36 Suppl 2:S113-9.

25. Schwartz SS, et al.The Time Is Right for a New Classification System for Diabetes: Rationale and Implications of the $\beta$-Cell-Centric Classification Schema.Diabetes Care 2016;39:179-86.

26. Wang P, et al.Effects of a Novel Glucokinase Activator, HMS5552, on Glucose Metabolism in a Rat Model of Type 2 Diabetes Mellitus.J Diabetes Res 2017;2017:5812607.

27. Matthews DR, et al.Glycaemic durability of an early combination therapy with vildagliptin and metformin versus sequential metformin monotherapy in newly diagnosed type 2 diabetes (VERIFY): a 5-year, multicentre, randomised, double-blind trial.Lancet 2019;394:1519-1529.

\section{Figures}


a.

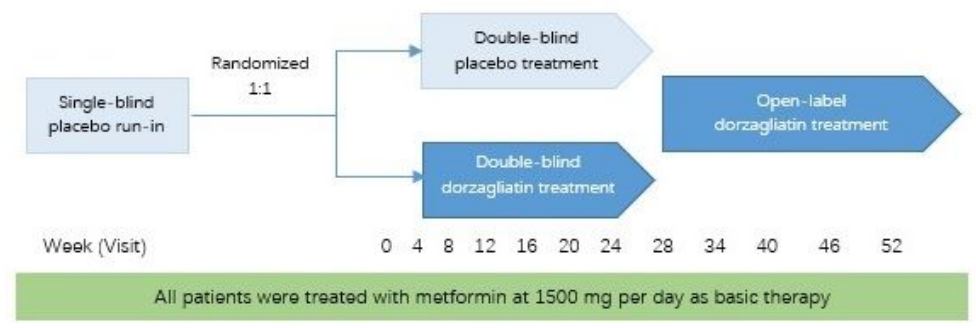

b.

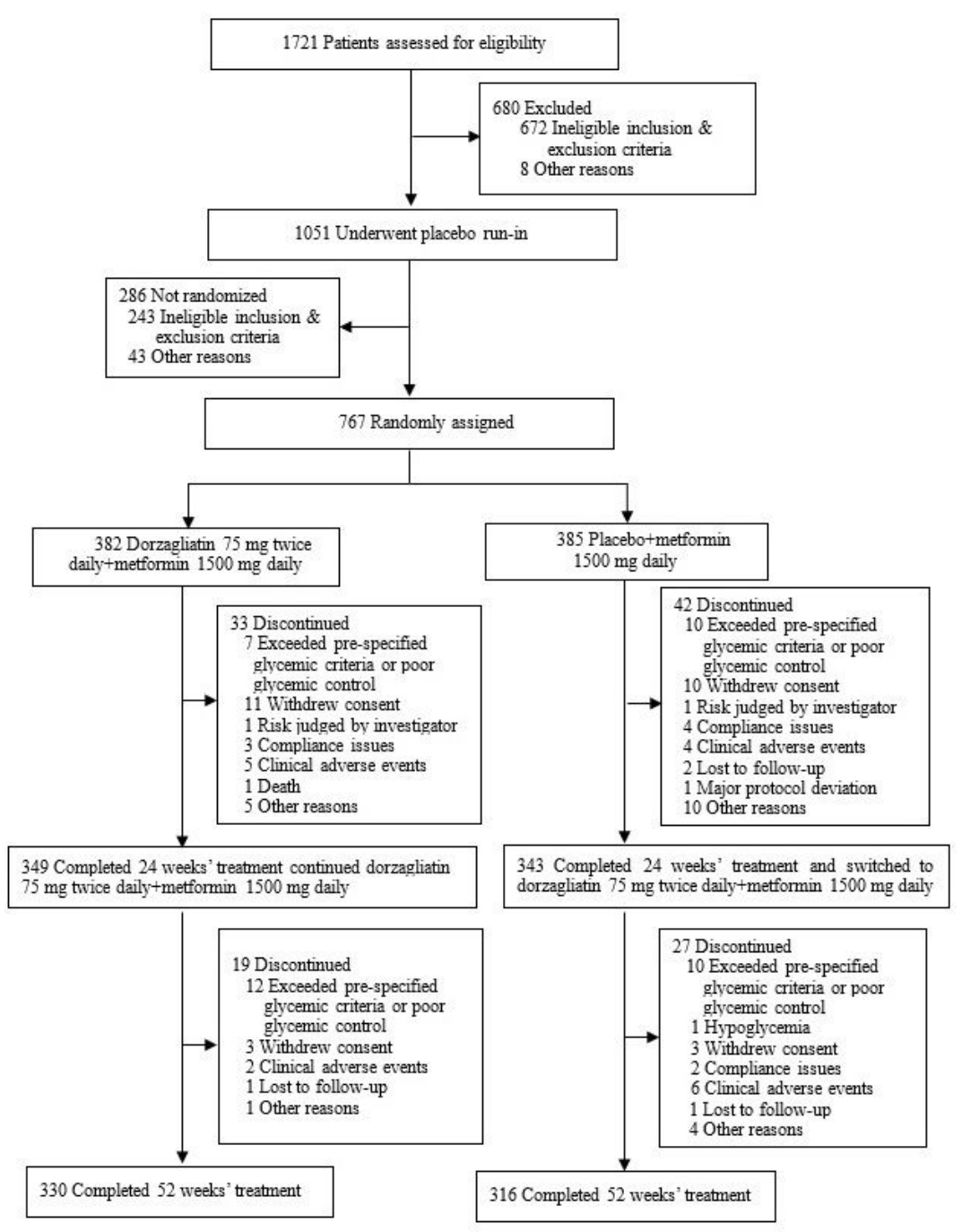

\section{Figure 1}

DAWN study design and study patient disposition. a. DAWN trial design. b. DAWN study patient disposition flow diagram throughout the trial.. Note: Ten patients did not meet the inclusion \& exclusion criteria but were admitted in the run-in period. Two patients did not meet the criteria for randomization but were randomized. 767 patients were randomized and 766 patients took at least one dose of study drug 

Eneragliatin+Mctformin
$\rightarrow$ Dorzagliatin+Metformin
- Placebo-Metformin
- MacebotMetformin
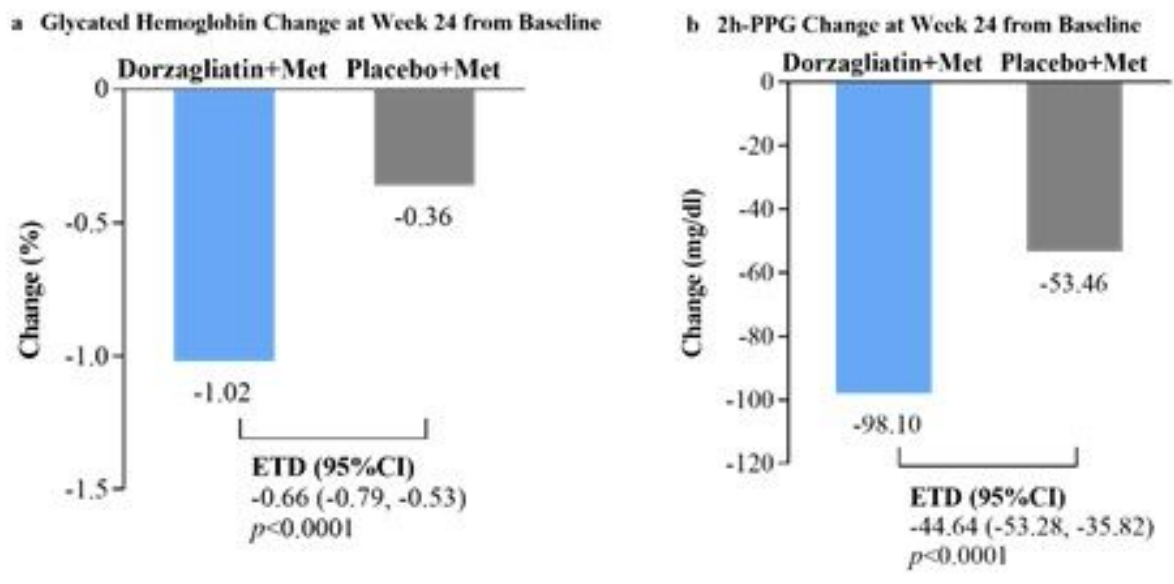

c Composite End Point of Respense Rate at Weck 24

d HOML2- $\beta$ Change at Week 24 from Baseline
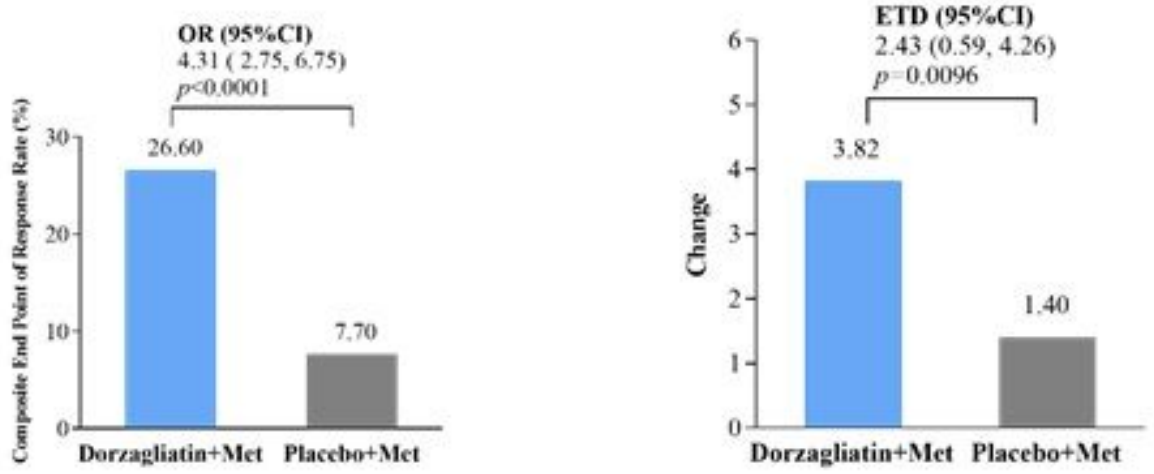

e HOMA2-IR Change at Week 24 from Baseline

f Glycated Hemoglobin Since Randomiration
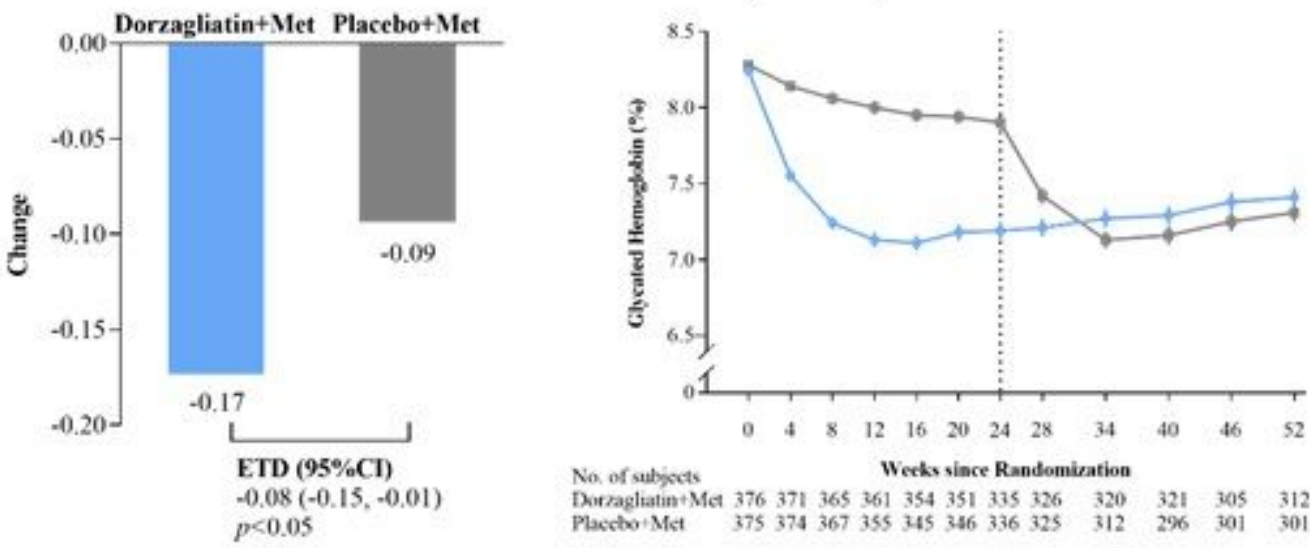

Figure 2

Primary, Secondary and Other Efficacy Endpoints*. a and b show the least-squares (LS) mean change from baseline in the glycated hemoglobin level (a) and 2-hour postprandial glucose level (b) at week 24. Estimated treatment difference (ETD) and corresponding 95\% confidence interval (Cl) were estimated using a mixed model for repeated measures in the full-analysis set. The full-analysis set comprised all randomized patients, who took at least one dose of study drugs and had at least one post-treatment measurement of the primary endpoint during double-blind treatment period (16 randomized patients were excluded from the full-analysis set: 15 owing to lack of efficacy assessments and 1 owing to no drugs 
taken after randomization). c shows the composite endpoint of response rate (glycated hemoglobin $<7.0 \%$, without hypoglycemia events and no weight gain) at week 24 . The rate was estimated in the fullanalysis set with the use of the data imputed by last observation carried forward. Logistic regression was used to estimate the odds ratio and $95 \% \mathrm{Cl}$ between the two treatment groups. $d$ and e show the LS mean change from baseline in the homeostasis model assessment 2- $\beta$ (d) and homeostasis model assessment 2-IR (e) at week 24 estimated from an analysis of covariance model in the full-analysis set, respectively. Homeostasis model assessment 2- $\beta$ and homeostasis model assessment 2-IR are indexes for evaluating pancreatic $\beta$-cell function and insulin resistance with the updated homeostasis model, respectively. They are developed from homeostasis model assessment 1 , including physiological indicators of glucose homeostasis such as liver and peripheral insulin resistance. f shows the arithmetic mean change in the glycated hemoglobin level over 52 weeks in the full-analysis set. Error bars indicate standard errors. * Metformin 1500 mg per day were given to each group as basic treatment. Met: metformin; ETD: estimated treatment difference; Cl: confidence interval; 2h-PPG: 2-hour postprandial glucose; OR: odds ratio; HOMA2: homeostasis model assessment 2.

\section{Supplementary Files}

This is a list of supplementary files associated with this preprint. Click to download.

- DAWNsupplementarytables.docx

- DAWNstudymanuscriptsupplement.pdf 